\title{
Initial conditions and charged multiplicities in ultra-relativistic heavy-ion collisions
}

\author{
Alberto Accardi \\ Dipartimento di Fisica Teorica, Università di Trieste, \\ Strada Costiera 11, I-34014 Trieste \\ and \\ INFN, Sezione di Trieste \\ via Valerio 2, I-34127 Trieste
}

\begin{abstract}
At ultra-relativistic energies the minijet production in heavy-ion collisions becomes sensitive to semi-hard parton rescatterings in the initial stages of the process. As a result global characteristics of the event, like the initial minijet density, become rather insensitive on the infrared cutoff that separates hard and soft interactions. This allows to define a nearly parameter-free saturation cutoff at which the initial conditions may be computed. As an application we study the centrality dependence of the charged particle multiplicity, which is compared with present RHIC data and predicted at higher energies.
\end{abstract}

PACS: 11.80.La, 24.85.+p, 25.75.-q

\footnotetext{
${ }^{1}$ E-mail:accardi@ts.infn.it
} 


\section{Introduction}

In heavy-ion collisions the partonic degrees of freedom of the two interacting nuclei become more and more important as the center of mass energy of the collision increases. At some point the main particle production mechanism in the initial stage becomes the liberation from the nuclear wave functions of a great number of partons, also called minijet plasma. At ultra-relativistic energies the partonic density of the two nuclei is so high that perturbative methods on one hand [1-6] and semi-classical non-perturbative methods on the other [7] become applicable to the computation of the initial conditions of the minijet plasma. It's successive evolution will possibly lead to thermalization of the system and to the transition to the quark-gluon plasma phase, whose formation and characteristics depend crucially on such initial conditions. Though the latter are not directly accessible experimentally, they can be related to final state observables, like the charged particle multiplicity and transverse energy, allowing a test of the proposed theoretical models.

We can divide in general the models in three classes: i) two-component models [5, 6], in which particle production is assumed to be decomposable into the sum of a soft and a hard part according to some cutoff $p_{0}$; ii) saturation models [1, 3, [4, 4], which exploit the high parton densities involved in the process; iii) "others", like the Dual Parton Model [8] and hydrodynamic models [9]. To distinguish between them, it has been proposed in [5] to study the centrality dependence of the charged particle multiplicity, since this allows to disentangle to some degree the dynamical and the geometrical effects. For a review of the results of the above models on the charged multiplicity see [10].

At very high energies the target parton densities experienced by projectile partons are so high that the probability for them to have more than one semi-hard scattering may become non negligible already at the BNL Relativistic Heavy-Ion Collider (RHIC). At such regimes the usual perturbative computation [2], obtained by eikonalization of the minijet cross-section, may become inadequate. Indeed, it takes into account only disconnected two-parton interactions located at different points in transverse space but neglects the rescatterings. With the help of a few simplifying hypotheses semi-hard parton rescatterings have been included in the interaction mechanism in [11, 12, and lead to sizeable effects already at RHIC energies [13] 15. Based on these results, in this paper we propose a new saturation mechanism for semi-hard minijet production and use it in a two-component model to compute charged particle multiplicities at RHIC and at the CERN Large Hadron Collider (LHC).

\section{Initial conditions and saturation}

When rescatterings are included in the interaction of two nuclei of atomic numbers $\mathrm{A}$ and $\mathrm{B}$, the average number of $\mathrm{A}$ nucleus minijets at fixed impact parameter $b$ is given by 11$]$ :

$$
N_{A}^{m j}(b)=\int d^{2} r d x \Gamma_{A}(x, b-r)\left[1-e^{-k \int d x^{\prime} \sigma_{H}\left(x x^{\prime}\right) \Gamma_{B}\left(x^{\prime}, r\right)}\right]
$$

and the average minijet initial multiplicity is obtained by summing the analogous contri-

bution from the B nucleus, $N^{m j}=N_{A}^{m j}+N_{B}^{m j}$. For simplicity we omit the flavour indices 
and consider only gluon-gluon interactions in our formulae, the inclusion of quarks being straightforward. In the numerical computations both the gluons and the quarks have been included. In Eq. (2.1), $\Gamma_{A}=\tau_{A}(r) G(x)$ is the nuclear parton distribution function of the A nucleus, $\tau_{A}(r)$ is its nuclear thickness function, normalized to A, evaluated at a transverse coordinate $r$ relative to the center of the nucleus and $G(x)$ is the parton distribution function of a proton at a given fractional momentum $x$. For simplicity we omit the flavour indices. $\sigma_{H}$ is the pQCD gluon-gluon cross-section at leading order in the high energy limit,

$$
\sigma_{H}\left(x x^{\prime}\right)=\frac{9}{2} \pi \alpha_{s}^{2} \frac{1}{p_{0}^{2}}\left(1-\frac{4 p_{0}^{2}}{x x^{\prime} s}\right) \theta\left(x x^{\prime} s-4 p_{0}^{2}\right) \theta(1-x) \theta\left(1-x^{\prime}\right),
$$

where we included all the kinematic limits and $p_{0}$ is the cut-off that discriminates between soft and semi-hard interactions. We also included explicitly the $k$-factor, $k$, to take into account higher order corrections. Both the cross section and the parton distributions depend on a scale $Q=p_{0}$, which we take equal to the cutoff. In the numerical computations we will set $k=2$ and use Woods-Saxon thickness function and GRV98LO parton distribution functions 16 .

Eq. (2.1) may be interpreted as the integral of the average density of projectile partons (at a given $x$ and $r$ ) times the probability of having at least one semi-hard scattering against the target. The exponent in Eq. (2.1) may be interpreted as the opacity of the target nucleus, being proportional to the total transverse area occupied by its partons at the resolution scale $p_{0}$. Two interesting limiting cases may be studied. At high values of $p_{0}$ the target has a small opacity and is seen by the incoming partons as a rather dilute system. As a consequence $N^{m j} \approx 2 \int d^{2} r d x d x^{\prime} \Gamma_{A}(x, b-r) \sigma_{H}\left(x x^{\prime}\right) \Gamma_{B}\left(x^{\prime}\right)$, and we recover the usual perturbative result [2]. On the other hand, at low values of $p_{0}$ the target opacity increases: the target is becoming black to the projectile partons. As a consequence, the probability of scattering at least once becomes so high that nearly every projectile parton scatters and the minijet multiplicity reaches a limiting value instead of diverging as it happens in the Eikonal computation.

In the regime where the target is almost black the semi-hard interactions are extracting from the projectile nucleus wave-function all its partons, and even if we use a lower cutoff no more partons are there to be extracted. for this reason the minijet multiplicity tends to saturate [13], see also Fig. 17a. We call saturation cutoff the value of $p_{0}$ at which this happens, and will denote it as $p_{\text {sat }}$. Of course the validity of this picture is limited to the kinematic regions where the saturation cutoff is in the perturbative range, $p_{\text {sat }} \gg \Lambda_{Q C D}$. To give a quantitative definition of the saturation cutoff we start by considering a central collision of two equal nuclei. We define the upper bound for the minijet multiplicity as

$$
N_{A l i m}^{m j}(b=0)=\lim _{k \rightarrow \infty} N_{A}^{m j}=\int_{4 p_{0}^{2} / s \leq x \leq 1} d^{2} r d x \Gamma_{A}(x, r) .
$$

Taking a very large $k$-factor corresponds, indeed, to the limit in which the target becomes completely black and the semi-hard interactions are effective in extracting all the partons from the projectile nucleus. The limiting procedure is needed in order to keep track of the kinematic limits. As it is easy to see, $N^{m j} \underset{p_{0} \rightarrow 0}{\sim} N_{l i m}^{m j}$, therefore we can define the saturation 

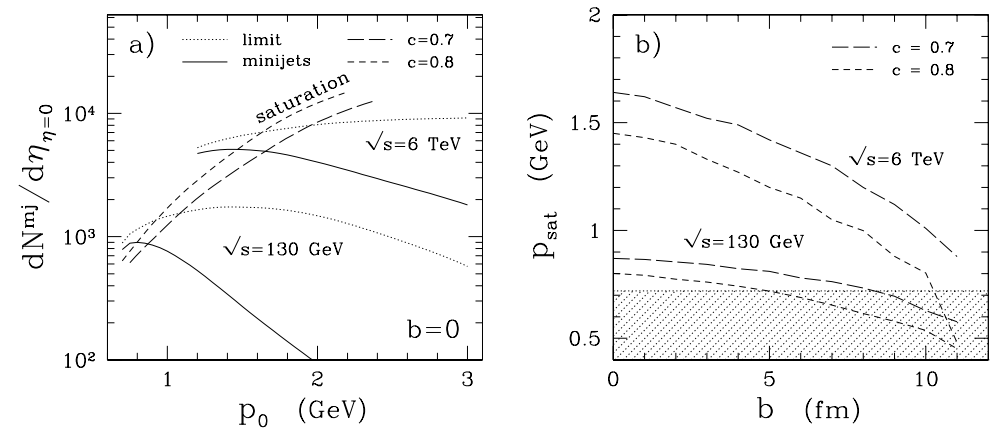

Figure 1: a) The minijet multiplicity, $N^{m j}$ (solid line), and its limiting value, $N_{\text {lim }}^{m j}$ (dotted line), in a central $\mathrm{Au}-\mathrm{Au}$ collision as a function of the cutoff $p_{0}$ at RHIC and LHC energies. The dashed lines are the saturated minijet multiplicities, $N_{\text {sat }}^{m j}$, with a saturation parameter $c=0.7$ (long dashes) and $c=0.8$ (short dashes). The intercept of the dashed lines with the dotted lines determines the saturation cutoff. b) The saturation cutoff as a function of the impact parameter at RHIC and LHC energies. The shaded area is the region where we estimate that the saturation criteria ceases to be valid.

cutoff as the value of $p_{0}$ such that the minijet multiplicity becomes a substantial fraction of its limiting value:

$$
N^{m j}\left(p_{0}=p_{\text {sat }}\right)=c N_{\text {lim }}^{m j}\left(p_{0}=p_{\text {sat }}\right)
$$

where the saturation parameter, $c$, is a positive number smaller than one. Notice that $p_{\text {sat }}=p_{\text {sat }}(\sqrt{s}, c)$ is a function also of the energy of the collision. From our discussion it is obvious that $c$ must be close to one to let $p_{\text {sat }}$ lie in the region where $N^{m j}$ is saturating. However, to stay in the perturbative regime we cannot choose it too close to one since $p_{\text {sat }} \rightarrow 0$ as $c \rightarrow 1$. Finally, we define the saturated minijet multiplicity as the average multiplicity evaluated at the saturation cutoff:

$$
N_{s a t}^{m j}=N_{s a t}^{m j}(\sqrt{s}, c)=N^{m j}\left(p_{0}=p_{s a t}\right)
$$

In our approach this number represents also the multiplicity of partons produced in the early stage of the heavy ion collision.

In Fig. 17a we show the minijet multiplicity and its limiting value as a function of the cutoff $p_{0}$ at RHIC and LHC energies. The rapidity density at $\eta=0$ is computed by integrating Eqs. (2.1) and (2.2) over a pseudo-rapidity interval $|\eta| \leq 1$, where we approximated $\eta \approx \log \left(x \sqrt{s} / p_{0}\right)$, and by dividing the result by a factor two. The dashed lines represent the saturated initial conditions computed with $c=0.7$ and $c=0.8$. We can see that at a given energy $N_{\text {sat }}^{m j}$, which is obtained as the intercept of the solid and dashed lines, is nearly independent of the saturation parameter as long as the latter is close enough to one. Indeed, both at RHIC and LHC energy we obtain approximately a $3 \%$ increase in the saturated multiplicity going from $c=0.7$ and $c=0.8$. Therefore, whereas $c$ is an arbitrary parameter its actual choice doesn't affect strongly the determination of the initial conditions. The dependence of $p_{\text {sat }}$ on $\sqrt{s}$ was studied in [14] where it is shown that for central collisions the saturation criterion is applicable from RHIC energies on.

Unless we use nuclear thickness functions with sharp edges, like the hard-sphere distributions, by applying blindly the saturation criteria to non central collisions we would 
obtain an impact parameter independent bound on the minijet multiplicity. Indeed we would have $N_{l i m}^{m j}(b)=\int d^{2} r d x \Gamma_{A}(x, b-r)=\int d^{2} r d x \Gamma_{A}(x, r)$. In this way, by requiring saturation as in Eq. (2.3) we would be asking the semi-hard interactions to extract all the partons from the projectile nucleus even in a very peripheral region, which is clearly unphysical. A simple way to implement the collision geometry in the saturation criterion is to cut by hand the thickness functions outside a given radius $R_{c}$ of the order of the nuclear radius. However, the minijet multiplicity as a function of the centrality of the collision turns out to depend too strongly on the choice of $R_{c}$ except at very high centrality or very high energies [14].

To find a less arbitrary way of implementing the collision geometry we look at the Glauber model computation of the average number of nucleons which participate in the collision:

$$
N_{\text {part }}(b)=\int d^{2} r \tau_{A}(b-r) \mathcal{P}_{B}(r)+A \leftrightarrow B
$$

where $\mathcal{P}_{B}(r)=1-\left[1-\sigma_{p p}(s) \tau_{B}(r) / B\right]^{B}$ and $\sigma_{p p}$ is the inelastic $p p$ cross-section, which we take from [17]. At $\sqrt{s}=130,200,6000 \mathrm{GeV}$ we have $\sigma_{p p}=39,42,75$ mbarn, respectively. $\mathcal{P}_{B}$ is the probability that a projectile nucleus at a given transverse coordinate $r$ has at least one inelastic interaction with the target nucleons. Then, we may require the saturation only for the fraction of projectile partons that belong to a participating nucleon, and define an effective nuclear distribution function

$$
\bar{\Gamma}_{A B}(x, b, r)=\Gamma_{A}(x, b-r) \mathcal{P}_{B}(r) .
$$

Correspondingly, we have an effective minijet multiplicity, $\bar{N}_{A}^{m j}(b)=\int d^{2} r d x \bar{\Gamma}_{A B}(x, b, r)[1-$ $\left.\exp \left(-\int d x^{\prime} \sigma_{H}\left(x x^{\prime}\right) \Gamma_{b}\left(x^{\prime}\right)\right)\right]$, and an effective upper limit, $\bar{N}_{A l i m}^{m j}(b)=\int d^{2} r d x \bar{\Gamma}_{A B}(x, b, r)$, which is no more $b$-independent. Then, the saturation criterion generalized to arbitrary impact parameter becomes:

$$
\bar{N}^{m j}\left(p_{0}=p_{\text {sat }}\right)=c \bar{N}_{l i m}^{m j}\left(p_{0}=p_{\text {sat }}\right) .
$$

Finally, having determined $p_{\text {sat }}$ in this way we use it in Eq. (2.4) to compute the average initial parton multiplicity.

In Fig. 1 $1 \mathrm{~b}$ we show the saturation cutoff as a function of the impact parameter at RHIC and LHC energies for different saturation parameters $c$. The horizontal line show the limit of approximately $0.7 \mathrm{GeV}$ whose intersection with $p_{\text {sat }}(b)$ sets the limit of validity of the present approach, as will be discussed in the next section.

Notice that the saturation cutoff, and consequently the initial conditions, are practically determined by the choice of the parton distribution functions. As explained above and in [13] the initial conditions are nearly independent of the remaining free parameters, namely the saturation parameter c and the $k$-factor.

In the proposed mechanism saturation is reached when there are no more partons that semi-hard interactions can extract from the nuclear wave functions. In this sense this mechanism is a saturation of the minijet production and is intermediate between initial and final state saturation. In initial state saturation [1] (see also [6, 7]) the parton 


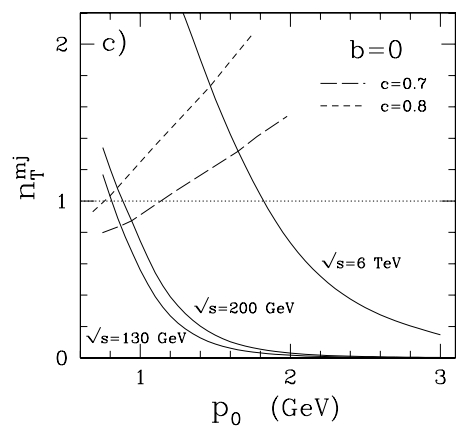

Figure 2: The minijet average occupation number in the transverse area (solid lines): $n_{T}^{m j}=A_{T}^{m j} / A_{T}^{A u}$, where $A_{T}^{m j}=N^{m j}\left(p_{0}\right) \times \pi / p_{0}^{2}$ and $A_{T}^{A u}$ is the transverse area of a gold nucleus. The dashed lines show the average occupation number of the saturated minijets as a function of the saturation momentum. When $n_{T}^{m j} \gtrsim 1$ the minijets begin to overlap transversely.

density inside the incoming nuclei saturate due to a compensation between parton splitting and parton fusion processes in the DGLAP evolution, which induces a corresponding saturation in the minijet multiplicity. On the contrary, in the final state mechanism [3, [4] the saturation is assumed to be caused by the high density of produced minijets, which screens softer parton production due to parton fusion processes in the final state. In particular these final state interactions are assumed to set in when the transverse area occupied by the minijets becomes comparable to the nuclear overlap area. Both processes may, therefore, complement our saturation mechanism since the former modify the input parton distribution functions and the latter deals with a later stage process. However, as we can see in Fig. 22, at $\sqrt{s}=130 \mathrm{GeV}$ and $\sqrt{s}=200 \mathrm{GeV}$ the saturated minijets fill the transverse area only partially. Therefore final state saturation effects should not alter significantly our computations at RHIC energy, but may play some role at LHC.

\section{Charged particle multiplicity}

We want to apply the saturation criterion for the semi-hard parton production in the initial stage of the collision to the computation of the charged particle multiplicity. Thanks to the self-shadowing property of the semi-hard interactions [15, 18], even if in Eq. (2.1) only the semi-hard cross-section, $\sigma_{H}$, appears, we are actually taking into account all the partons that had at least one semi-hard scattering, while their other scatterings may be semi-hard or soft with no restrictions. Therefore we are missing only the purely soft part of the production mechanism. This leads us to adopt a two-component model in which the charged particle multiplicity is written as the sum of a soft and a semi-hard part: $d N^{c h} / d \eta(b)=d N_{\text {soft }}^{c h} / d \eta(b)+d N_{s . h .}^{c h} / d \eta(b)$. The soft part is assumed to scale with the number of participants, Eq. (2.5), so that [6]

$$
\frac{d N_{s o f t}^{c h}}{d \eta}(b)=x n_{p \bar{p}}(s) \frac{N_{\text {part }}(b)}{2} .
$$

Here $n_{p \bar{p}}(s)$ the pseudo-rapidity density of charged particles produced at $\eta=0$ in $p \bar{p}$ collision at a given c.m. energy $\sqrt{s}$. We use the fit [19], $n_{p \bar{p}}(s)=2.5-0.25 \log (s)+0.023 \log ^{2}(s)$. 
The coefficient $x=x(s)$ is a parameter that allows to adjust the relative weight of soft and semi-hard interactions and will be determined from the experimental data. Further, we assume the semi-hard part to be completely computable from the saturation criterion for minijet production described in the last section. To convert the minijet multiplicity to charged particle multiplicity, we further assume isentropic expansion of the initially produced minijet plasma and parton-hadron duality, so that

$$
\frac{d N_{s . h .}^{c h}}{d \eta}(b)=0.9 \times \frac{2}{3} \times \frac{d N_{s a t}^{m j}}{d \eta}(b),
$$

where the factor 0.9 is due to the different number of degrees of freedom of the system in the minijet-plasma phase and in the hadronic phase [3]. To mark out the contribution of the hard part it is customary to divide the charged multiplicity by the number of participant pairs, so that the observables we are interested in are:

$$
\frac{1}{N_{\text {part }}(b) / 2} \frac{d N^{c h}}{d \eta}(b)=x n_{p p}(s)+\frac{1}{N_{\text {part }}(b) / 2} \frac{d N_{s . h .}^{c h}}{d \eta}(b)
$$

and the fraction of semi-hard interactions, $F_{s . h .}=\frac{d N_{s . h .}^{c h}}{d \eta} / \frac{d N^{c h}}{d \eta}$.

To make a comparison with experimental data we have first to relate the observables appearing in Eq. (3.3), which are functions of the impact parameter, to the experimental ones, which are obtained as averages over centrality classes of events [20 22]. Following [6, 23., to which we refer for the details, we do this by studying the minimum bias multiplicity distribution of charged particles and by dividing the events in suitable subsets over which the average is performed. The next step is to extract the parameter $x$ in Eq. (3.1) by comparing the computation for the $3 \%$ most central events and the PHOBOs data at $\sqrt{s}=130 \mathrm{GeV}$ from Ref. [21]. This value is then used to make predictions at higher energy.

In Fig. 3 we show both the results for the semi-hard part before the averaging over the centrality classes, and the results obtained after the averaging and the inclusion of the soft part. For each curve the result obtained by setting $c=0.7$ and $c=0.8$ in Eq. (2.6) is shown.

At $\sqrt{s}=130 \mathrm{GeV}$ we find $x=0.445$ and $x=0.453$, for a saturation parameter $c=0.7$ and $c=0.8$, respectively. These values of $x$ correspond to a fraction of semi-hard interactions $F_{s . h}=0.805$ and $F_{s . h}=0.817$, respectively, and show a good stability with respect to $c$. The relatively large value of $F_{s . h}$. with respect to the common expectation of nearly a half and to the value of 0.37 extracted from PHoBos data in Ref. [6] is due to the fact that we considered as belonging to the non-soft part of the observable also a semi-hard region $0.7 \mathrm{GeV} \lesssim p_{0} \lesssim 2 \mathrm{GeV}$. Note that we can push our perturbative computations to such low values of the cutoff because inclusion of parton rescatterings results in a rather small sensitivity of global observables on $p_{0}$ in that region [13, 14].

The two curves start with a moderate slope at high centrality and at some point they decrease very fast. This happens when the corresponding saturation cutoff becomes smaller than $0.7 \mathrm{GeV}$, approximately. The reason for this behaviour is that the distribution functions are fitted just down to a scale $Q \approx 0.9 \mathrm{GeV}$ and they are numerically extrapolated at lower scales. Below $0.7 \mathrm{GeV}$ the extrapolation gives an unnaturally fast decrease of 


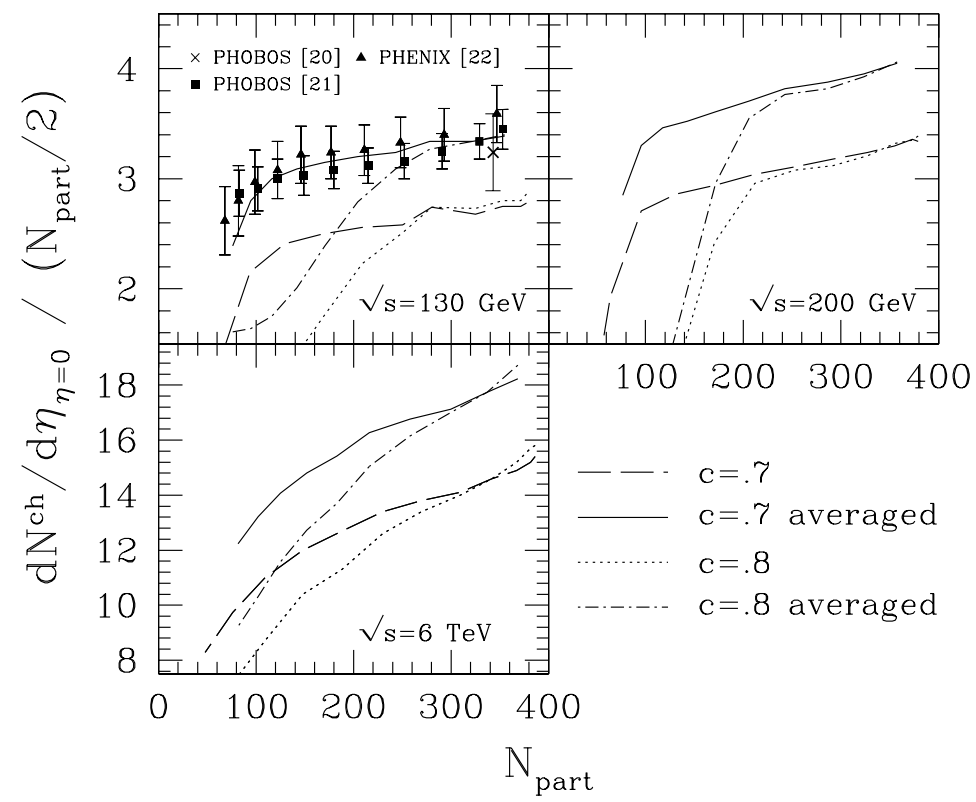

Figure 3: Charged particle multiplicity per participant pair, Eq. (3.3), as a function of the number of participants at different center of mass energies and saturation parameter $c=0.7$ and $c=0.8$. In each panel the lower pair of curves (dashed and dotted lines) represent the semi-hard contribution. The upper pair of curves (solid and dot-dashed lines) are obtained by averaging the semi-hard contribution and by adding the soft part. At $\sqrt{s}=130 \mathrm{GeV}$ the parameter $x$ is extracted from the $3 \%$ most central PHOBOs events at $\sqrt{s}=130 \mathrm{GeV}$ [21]. At higher energies the upper curves may be considered an upper bound, while the lower ones give a lower bound, see text.

the parton densities, which results in the rapid fall of the minijet production. Then, we define the region of validity of our computations as the one such that $p_{\text {sat }} \gtrsim 0.7 \mathrm{GeV}$, or in other words the one to the right of the knee in the charged multiplicity.

The value of $p_{\text {sat }}$ at fixed centrality decreases when the saturation parameter $c$ increases (see Fig. 1), therefore the curve with $c=0.8$ is reliable for a smaller range of centrality than the curve with $c=0.7$. They agree, however, in the common region of validity (showing a slight tendency to increase their slope when increasing $c$ ), and after the experimental averaging and the fit to the most central data point, both describe well the experimental data.

At $\sqrt{s}=200 \mathrm{GeV}$ we don't have any data to normalize the multiplicities to. However, the fraction of semi-hard to soft interactions is expected to grow with the energy of the collision, and we can use the value of $F_{s . h}$. determined at $\sqrt{s}=130 \mathrm{GeV}$ to obtain an approximate upper bound for the charged multiplicities: for $\sqrt{s} \geq 130$

$$
x n_{p \bar{p}}(s) \leq \frac{1-\left.F_{s . h .}\right|_{b=0, \sqrt{s}=130 \mathrm{GeV}}}{\left.F_{s . h .}\right|_{b=0, \sqrt{s}=130 \mathrm{GeV}}} \frac{d N_{s . h .}^{c h} / d \eta}{N_{\text {part }} / 2}(b=0, s) .
$$

The curves for the two values of $c$ agree over a wider range of neutralities. This is to be expected since the saturation cutoff at fixed centrality grows with the center of mass energy, and goes below the critical value of $0.7 \mathrm{GeV}$ at smaller centrality. Notice also that 
the slope of the curves has increased.

At LHC energy, $\sqrt{s}=6 \mathrm{TeV}$, the particle production is generally believed to be almost completely semi-hard. Therefore we expect that the data will be close to the averaged semi-hard multiplicity without any normalization (which is very similar to the lower curve plotted in Fig. 3). Though the saturation criterion is applicable over all the centrality range considered (see Fig. 1 $1 \mathrm{~b}$ ) the slope of the curves is rather sensitive to the saturation parameter, resulting in a larger theoretical uncertainty. We expect that a better treatment of the scale $Q$ and of the pseudo-rapidity, which are taken to depend simply on the cutoff $p_{0}$, could solve at least partially this problem. However, the average slope has increased confirming the trend observed at lower energies.

\section{Conclusions}

The inclusion of semi-hard parton rescatterings in the interaction dynamics of heavyion collisions at very high energy allows a reliable computation of the initial conditions, like the minijet multiplicity, and the introduction of a nearly parameter-free saturation criterion to determine the infrared cutoff to be used in the perturbative computations. The proposed saturation mechanism is intermediate between the initial and final state ones in that it deals with the saturation in the production of minijets.

We tested our approach against RHIC data on the centrality dependence of charged multiplicities by using a two-component model in which the semi-hard part is assumed to be completely given by the proposed saturation criterion. At $\sqrt{s}=130 \mathrm{GeV}$ we find a good agreement with the data, which allows us to extrapolate the results at the highest RHIC energy of $\sqrt{s}=200 \mathrm{GeV}$ and at LHC energy, $\sqrt{s}=6 \mathrm{TeV}$, by putting upper and lower bounds on the charged multiplicities per participant pairs as a function of the number of participants and by predicting their slope.

\section{Acknowledgments}

I am very grateful to D. Treleani and P. Huovinen for their stimulating comments and a critical reading of the manuscript. I would like also to thank R. Arnaldi, M. Gyulassy, D. Kharzeev, H. J. Pirner, A. Polleri, C. Salgado U. Wiedemann and F. Yuan for many useful discussions. This work was partially supported by the Italian Ministry of the University and of the Scientific and Technological Research (MURST) by Grant No. COFIN99.

\section{References}

[1] L. V. Gribov, E. M. Levin and M. G. Ryskin, Phys. Rept. 100 (1983) 1; J. P. Blaizot and A. H. Mueller, Nucl. Phys. B289 (1987) 847; A. H. Mueller and J. Qiu, Nucl. Phys. B 268 (1986) 427; 
[2] K. Kajantie, P. V. Landshoff and J. Lindfors, Phys. Rev. Lett. 59 (1987) 2527.

[3] K. J. Eskola, K. Kajantie, P. V. Ruuskanen and K. Tuominen, Nucl. Phys. B 570 (2000) 379 .

[4] K. J. Eskola, K. Kajantie and K. Tuominen, Phys. Lett. B 497 (2001) 39; H. J. Pirner and F. Yuan, Phys. Lett. B 512 (2001) 297; K. J. Eskola, P. V. Ruuskanen, S. S. Rasanen and K. Tuominen, hep-ph/0104010; K. J. Eskola, K. Kajantie and K. Tuominen, hep-ph/0106330.

[5] X. N. Wang and M. Gyulassy, Phys. Rev. Lett. 86 (2001) 3496

[6] D. Kharzeev and M. Nardi, Phys. Lett. B 507 (2001) 121.

[7] L. McLerran, hep-ph/0104285 and references therein; A. H. Mueller, Nucl. Phys. B 572 (2000) 227.

[8] A. Capella and D. Sousa, Phys. Lett. B 511 (2001) 185.

[9] P. F. Kolb, U. Heinz, P. Huovinen, K. J. Eskola and K. Tuominen, hep-ph/0103234, and references therein.

[10] K. J. Eskola, hep-ph/0104058; M. Gyulassy, nucl-th/0106072.

[11] G. Calucci and D. Treleani, Phys. Rev. D41, 3367 (1990);

[12] G. Calucci and D. Treleani, Phys. Rev. D44, 2746 (1991); Int. J. Mod. Phys. A 6 (1991) 4375; Phys. Rev. D 49 (1994) 138; Phys. Rev. D 50 (1994) 4703.

[13] A. Accardi and D. Treleani, Phys. Rev. D 63 (2001) 116002.

[14] A. Accardi, hep-ph/0104060.

[15] A. Accardi and D. Treleani, hep-ph/0106306.

[16] M. Gluck, E. Reya and A. Vogt, Eur. Phys. J. C5 (1998) 461;

[17] The Review of Particle Physics, Eur. Phys. Jou. C15 (2000) 1, Fig. 37.19

[18] R. Blankenbecler, A. Capella, C. Pajares, J. Tran Thanh Van and A. Ramallo, Phys. Lett. B 107 (1981) 106; C. Pajares and A. V. Ramallo, Phys. Rev. D 31 (1985) 2800; D. Treleani, Int. J. Mod. Phys. A 11 (1996) 613.

[19] F. Abe et al. [CDF Collaboration],, Phys. Rev. D 41 (1990) 2330.

[20] B. B. Back et al. [PHOBOS Collaboration], Phys. Rev. Lett. 85 (2000) 3100.

[21] B. B. Back et al. [PHOBOS Collaboration], nucl-ex/0105011.

[22] K. Adcox et al. [PHENIX Collaboration], Phys. Rev. Lett. 86 (2001) 3500.

[23] D. Kharzeev, C. Lourenco, M. Nardi and H. Satz, Z. Phys. C 74 (1997) 307. 